\title{
MODELANDO O ESCOAMENTO ATMOSFÉRICO NOTURNO EM UMA REGIÃO DE FLORESTA
}

\author{
Pablo E. S. Oliveira ${ }^{1}$, Felipe D. Costa ${ }^{2}$, Otavio C. Acevedo ${ }^{1}$ \\ ${ }^{1}$ Universidade Federal de Santa Maria \\ ${ }^{2}$ Universidade Federal do Pampa \\ pablo@ufsm.br
}

\section{RESUMO}

Neste trabalho, um modelo simplificado é utilizado para simular o comportamento da turbulência na camada limite noturna em uma região de floresta. Apesar das simplificações inicialmente impostas, o modelo é capaz de reproduzir alguns aspectos do escoamento atmosférico, em condições estáveis, tais como o acoplamento atmosférico e a intermitência.

\begin{abstract}
In this work a simplified model is used to simulate the turbulence behavior in the nocturnal boundary layer in a forest region. In spite of the simplifications, the model is able to reproduce some aspects atmospheric flow, under stable conditions, like the atmospheric coupling and the global intermittency.
\end{abstract}

\section{INTRODUÇÃO}

Um dos principais mecanismos responsáveis por afetar as estimativas de fluxos noturnos é a ocorrência de intermitência, em condições muito estáveis. Além disso, uma importante questão a ser considerada refere-se ao acoplamento dinâmico que ocorre entre os diferentes níveis atmosféricos, e em especial para o $\mathrm{CO}_{2}$, já que uma substancial porção da emissão noturna desse escalar ocorre devido à respiração no solo e plantas próximas ao solo, tornando assim, muito importante o entendimento dos processos físicos que ocorrem nestas condições. Assim, neste trabalho, é utilizado um modelo simplificado para estudar o comportamento do escoamento atmosférico em condições muito estáveis, em uma região de floresta. 


\section{MATERIAIS E MÉTODOS}

a. Modelo

Em uma situação idealizada e estimando fluxos turbulentos em função da intensidade turbulenta, representada pela velocidade de fricção $\left(u_{*}^{2}=E / 5.5\right)$, as equações que descrevem o escoamento atmosférico na CLE, podem ser escritas como:

$$
\begin{gathered}
\frac{\partial u}{\partial t}=f\left(v-v_{G}\right)-\frac{\partial\left(u_{*}^{2} \cos \psi\right)}{\partial z} \\
\frac{\partial v}{\partial t}=f\left(u_{G}-u\right)-\frac{\partial\left(u_{*}^{2} \sin \psi\right)}{\partial z} \\
\frac{\partial \theta}{\partial t}=-\frac{\partial u_{*} \theta_{*}}{\partial z} \\
\frac{\partial E}{\partial t}=S u_{*}^{2}-R i S u_{*}^{2}+\left[K_{E} \frac{\partial E}{\partial Z}\right]-c_{\varepsilon} \frac{u_{*}^{3}}{l_{m}}
\end{gathered}
$$

onde $u, v$ e $u_{G}, v_{G}$ são as componentes do vento e do vento geostrófico, respectivamente, $f$ é o parâmetro de Coriolis em São João, PR, Brasil (25 34'18"S e 50 05'56"W), $\psi$ é direção do vento, $\theta_{*}=K_{h}(\partial \theta / \partial z)$ é a escala de temperatura, $S$ é o cisalhamento do vento, $R i$ é o número de Richardson. Por simplicidade, os coeficientes de difusão são parametrizados como: $K_{m}=\kappa u_{*} z ; K_{E}=K_{m} / \sigma_{E}$ e $K_{h}=K_{m} / \operatorname{Pr}$, sendo $\sigma_{E}=2,5$ e $\operatorname{Pr}=1$.

\section{b. Parametrização de Floresta e Discretização}

Os efeitos do dossel foram representados pela adição de um termo de arrasto (BAILEY E STOLL, 2013):

$$
D_{i}=C_{d} a \tilde{u}_{i} V
$$

onde $C_{d}$ é o coeficiente de arrasto, $a$ é a densidade de área foliar (DAF) e $V$ é a velocidade escalar do vento. O perfil vertical de DAF foi estimado conforme proposto em LALIC E MIHAILOVIC (2004). Já no interior da floresta, o perfil de temperatura foi assumido 
constante, durante toda a simulação, e igual a 290 K. É importante ressaltar que está é uma imposição inicial que deve ser parametrizada adequadamente no futuro.

As equações 1 - 4 foram discretizadas em um domínio vertical de 100 m, o qual é dividido em 4 níveis, igualmente espaçados entre o solo e a copa das árvores ( $h=25 \mathrm{~m}$ ) e acima do topo da floresta o domínio é divido em 6 níveis sendo que o primeiro é mantido fixo em $h+5$ m e os demais são igualmente espaçados. O conjunto de equações resultantes da discretização é integrado utilizando o método Runge-Kutta de quarta ordem.

\section{RESULTADOS}

A figura 1a mostra que quanto mais fraco é vento geostrófico $\left(u_{G}\right)$, mais rasa é a camada limite noturna, já que o vento tende a um valor constante em uma altura mais próxima do solo e a medida que o forçante mecânico aumenta a o domínio vai se tornando completamente turbulento. Todavia, é importante destacar que o atrito reduz significativamente a intensidade do vento no interior do dossel e consecutivamente a atividade turbulenta, como pode ser observado nos perfis verticais de ECT (fig. 1c), que mostram que a intensidade turbulenta no interior do dossel aumenta consideravelmente à medida que $u_{G}$ umenta. Todavia, a influência do dossel não é representada realisticamente nos perfis de temperatura (fig. 1b), já que a mesma é mantida constante no interior da floresta. Esta implicação leva a resultados irreais para valores elevados de $u_{G}$ já que a curvatura do perfil aumenta com o aumento da intensidade turbulenta.

É importante destacar que apesar das simplificações, o modelo é capaz reproduzir o acoplamento atmosférico (fig. 1d), e, além disso, que no estado desconectado é observada a ocorrência de eventos turbulentos de forma intermitente similarmente ao que ocorre no mundo real (fig. 1d).

\section{CONCLUSÃO}

Através de um modelo simplificado foi possível reproduzir de maneira qualitativa alguns aspectos reais do comportamento do escoamento atmosférico em regiões de floresta. É importante destacar que este é um trabalho inicial que pode evoluir em muitos aspectos 
como, por exemplo, a inclusão de uma taxa de variação de temperatura no interior e no topo do dossel.
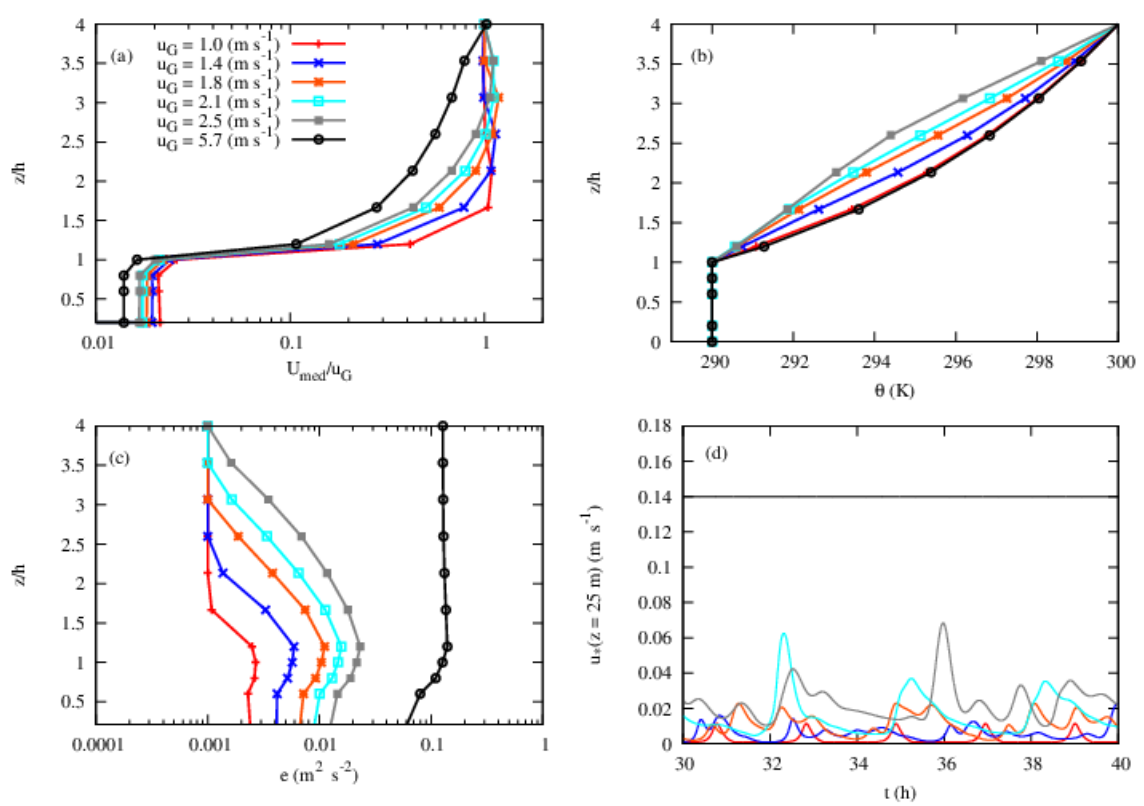

Figura 1: Perfis verticais de vento médio (normalizado por $u_{G}$ ) (fig. 1a), temperatura potencial (fig 1b) e ECT (fig. 1c) para diferentes valores do forçante mecânico do topo da CLE indicados pela legenda. As séries temporais de $u_{*}$ para cada caso apresentado nas figuras 1a - 1c, são mostradas na figura $1 \mathrm{~d}$.

\section{REFERÊNCIAS BIBLIOGRÁFICAS}

BAILEY, B. N.; STOLL, R. Turbulence in sparse, organized vegetative canopies: a largeeddy simulation study. Boundary-Layer Meteorology, v. 147, p. 369-400, 2013.

LALIC, B.; MIHAILOVIC, D. T. An empirical relation describing leaf-area density inside the forest for environmental modeling. Journal of Applied Meteorology, v. 43, p. 641-645, 2004. 